ANNALES

POLONICI MATHEMATICI

$81.3(2003)$

\title{
Propriétés d'extension et applications séparément holomorphes dans les espaces faiblement hyperboliques
}

\author{
par Omar Alehyane (El Jadida) et Hichame Amal (Rabat)
}

\begin{abstract}
The goal of this paper is to study the relationship between the hyperbolicity of complex spaces, extension of holomorphic mappings and the Hartogs theorem for separately holomorphic mappings. We prove that a complex space with a weak hyperbolicity which has the $\mathbb{D}^{*}$-extension property has the Hartogs extension property. As a consequence we give a generalization of the big Picard theorem. Finally we generalize Terada's theorem for separately holomorphic mappings.
\end{abstract}

1. Introduction. L'objectif de cet article est de dégager d'avantage les liens qui existent entre l'hyperbolicité des espaces analytiques, certaines propriétés d'extension d'applications holomorphes et le théorème de Hartogs pour les applications séparément holomorphes.

Dans la première section on établit que les espaces Kobayashi-hyperboliques, les variétés qui admettent une fonction strictement plurisousharmonique et les espaces holomorphiquement séparés de dimension pure appartiennent a une même famille d'espaces analytiques qu'on appellera espaces faiblement hyperboliques.

La seconde section est consacrée à la comparaison de la $\mathbb{D}^{*}$-extension et la propriété de Hartogs. Un espace analytique $X$ possède la propriété d'extension $\mathbb{D}^{*}\left(\mathbb{D}^{*}\right.$-EP) si pour toute application holomorphe $f$ de $\mathbb{D}^{*}$ dans $X$, il existe une application holomorphe $g \in \operatorname{Hol}(\mathbb{D}, X)$ telle que $g_{\mid \mathbb{D}^{*}}$ $=f$, où $\mathbb{D}=\{z \in \mathbb{C}|| z \mid<1\}$ et $\mathbb{D}^{*}=\mathbb{D} \backslash\{0\}$. Kwack [7] a montré que dans le cas où $X$ est compact, la $\mathbb{D}^{*}$-EP est équivalente à l'hyperbolicité au sens de Kobayashi; elle est aussi équivalente à l'hyperbolicité au sens de Brody [5]. Si $X$ n'est pas supposé compact, le résultat précédent n'est plus vrai ; $X=\mathbb{D}^{*}, f(z)=z$, donne un exemple de variété hyperbolique (même hyperbolique complète) qui n'a pas la propriété $\mathbb{D}^{*}$-EP. Mais si $X$ a la propriété $\mathbb{D}^{*}$-EP, alors $X$ est hyperbolique au sens de Brody [18].

2000 Mathematics Subject Classification: 32H02, 32H04, 32H25, 32Q45.

Key words and phrases: weak hyperbolic space, separately holomorphic map.

Ce travail a bénéficié du soutien matériel de l'Action Intégrée 180/MA/99 et de PARS MI 07. 
On dit que $X$ a la propriété de prolongement de Hartogs (PPH) si pour toute application holomorphe $f$ de la figure de Hartogs $H(r)$ à valeurs dans $X$, il existe $g \in \operatorname{Hol}\left(\mathbb{D}^{2}, X\right)$ telle que $g=f$ sur $H(r)$. Si $X$ est une variété de Stein, ou plus généralement $X$ est Kählerienne holomorphiquement convexe et ne possède pas de courbe rationnelle [4], alors $X$ a la propriété $(\mathrm{PPH})$.

Il est clair que la propriété $(\mathrm{PPH})$ n'implique pas toujours la $\mathbb{D}^{*}$-EP : en effet, $X=\mathbb{C}$ possède la ( $\mathrm{PPH})$ (c'est le théorème classique de Hartogs), mais $X$ n'a pas la $\mathbb{D}^{*}$-EP car $f(z)=1 / z$ pour $z \in \mathbb{D}^{*}$ n'admet pas de prolongement holomorphe à $\mathbb{D}$. Dans [20], Do Duc Thai et Nguyen Le Huong ont montré que si $X$ est pseudoconvexe, la $\mathbb{D}^{*}$-EP implique la (PPH). Dans la troisième section on démontre qu'un espace $X$ faiblement hyperbolique qui a la $\mathbb{D}^{*}$-EP a aussi la (PPH). Ce résultat sera utilisé dans la section suivante pour donner une généralisation du grand théorème de Picard.

Dans la dernière section on étudie le lien qui existe entre l'hyperbolicité et le théorème de Hartogs pour les applications séparément holomorphes. Le célèbre théorème de Hartogs sur l'analyticité des fonctions séparément holomorphes a fait l'objet de nombreux travaux, mais ce fût sans doute l'école japonaise avec notamment I. Shimoda [13] et T. Terada [17] qui a initié le problème de l'holomorphie des fonctions séparément holomorphes sur un produit d'ouverts en affaiblissant les hypothèses sur l'un des facteurs, obtenant ainsi une généralisation du théorème de Hartogs. Après Terada de nombreuses extensions du théorème de Hartogs pour les fonctions séparément holomorphes ont été données par Siciak ([15], [16]), Zahariuta [22], Shiffman [11], Nguyen Thanh Van et Zeriahi [8].

Le théorème de Hartogs n'est pas vrai pour les applications holomorphes dans les espaces analytiques générales. Comme exemple considérons l'application $f: \mathbb{C}^{2} \rightarrow \mathbb{P}_{1}$ définie par $f(z, w)=\left[(z+w)^{2}:(z-w)^{2}\right]$ si $(z, w) \neq(0,0)$ et $f(0,0)=[1: 1]$. On vérifie facilement que $f$ est séparément holomorphe sur $\mathbb{C} \times \mathbb{C}$, holomorphe sur $\mathbb{C}^{2} \backslash\{(0,0)\}$, mais n'est pas continue au point $(0,0)$.

B. Shiffman fût le premier, dans [12], à étendre certains résultats de Siciak et ceux de Terada aux applications séparément holomorphes à valeurs dans les espaces qui ont la propriété de prolongement de Hartogs. Dans [1] O. Alehyane a pu généraliser certains résultats de Shiffman, Nguyen Thanh Van et Zeriahi aux applications séparément holomorphes à valeurs dans ces mêmes espaces. Enfin Alehyane et Zeriahi [2] ont donné une autre version du théorème de Hartogs pour les applications séparément holomorphes entre espaces analytiques.

Dans la dernière section, on donne une généralisation du théorème de Hartogs pour les applications séparément holomorphes à valeurs dans les espaces faiblement hyperboliques. Dans le cas particulier des espaces hy- 
perboliques on donne une généralisation du théorème de Terada, et comme conséquence on montre le même résultat pour le cas des espaces holomorphiquement séparés de dimension pure.

2. Espaces faiblement hyperboliques. Soit $X$ un espace analytique complexe et notons par $d_{X}$ la pseudo-distance de Kobayashi de $X$.

DÉFInition 2.1. Un espace analytique $X$ est dit hyperbolique au sens de Kobayashi si $d_{X}$ est une distance $\operatorname{sur} X$, i.e. $d_{X}(x, y)>0$ si $x \neq y$. Cette distance induit alors la topologie de $X$.

Dans le cas où $X=\mathbb{D}$, cette pseudo-distance coïncide avec la métrique de Poincaré $: d_{\mathbb{D}}(z, w)=\varrho_{\mathbb{D}}(z, w)$.

Soient $X, Y$ deux espaces analytiques complexes, et $d_{X}$ et $d_{Y}$ les pseudodistances de Kobayashi de $X$ et $Y$ respectivement. Alors pour tout $f \in$ $\operatorname{Hol}(Y, X)$ on a

$$
d_{X}(f(x), f(y)) \leq d_{Y}(x, y) \quad \forall x, y \in Y .
$$

La proposition suivante, due à N. Sibony [14], donne une condition suffisante pour l'hyperbolicité :

Proposition 2.2 ([14]). Soit $X$ une variété analytique complexe admettant une fonction strictement plurisousharmonique négative. Alors $X$ est hyperbolique au sens de Kobayashi.

On dit qu'un espace analytique $X$ est Brody-hyperbolique si $X$ ne contient pas de courbe complexe, i.e. si toute application holomorphe $g \in \operatorname{Hol}(\mathbb{C}, X)$ est constante.

Tout espace analytique hyperbolique au sens de Kobayashi est Brodyhyperbolique, mais la réciproque est fausse en général.

DÉfinition 2.3. Soit $X$ un espace analytique complexe. On dit que $X$ est faiblement hyperbolique si pour tout compact $K \subset X$, il existe un voisinage ouvert $D$ de $K$ vérifiant :

(i) $D$ est hyperbolique,

(ii) pour tout $f \in \operatorname{Hol}(\mathbb{D}, X) \cap \mathcal{C}(\overline{\mathbb{D}}, X)$, si $f(\partial \mathbb{D}) \subset K$ alors $f(\overline{\mathbb{D}}) \subset D$.

ExEmPLES. 1) Tout espace hyperbolique est faiblement hyperbolique.

2) Si $X$ est une variété analytique admettant une fonction strictement plurisousharmonique, alors $X$ est faiblement hyperbolique. En effet, soit $u$ une fonction strictement Psh sur $X$ et soit $K \subset X$ un compact. Posons $v:=u-\left(\sup _{K} u+1\right)$ et $D:=\{x \in X \mid v(x)<0\}$. Alors $K \subset D$ et $\psi:=v_{\mid D}$ est négative strictement Psh sur $D$, donc $D$ est hyperbolique. Le principe du maximum permet de montrer (ii) de la définition 2.3.

3) Si $X$ est un revêtement ramifié d'un espace analytique $\widetilde{X}$ faiblement hyperbolique, alors $X$ est aussi faiblement hyperbolique. En effet, soit $\pi$ : 
$X \rightarrow \widetilde{X}$ et soit $K \subset X$ un compact. Alors $\widetilde{K}:=\pi(K)$ est compact dans $\widetilde{X}$, il existe donc un ouvert $\widetilde{D} \subset \widetilde{X}$ contenant $\widetilde{K}$ et vérifiant les propriétés (i) et (ii) de la définition 2.3. L'ouvert $D:=\pi^{-1}(\widetilde{D})$ contient $K$ et vérifie aussi les propriétés (i) et (ii) de la définition 2.3. Donc $X$ est faiblement hyperbolique.

4) L'espace projectif $\mathbb{P}_{1}$ n'est pas faiblement hyperbolique.

Remarque. Si $X$ est un espace analytique compact, alors $X$ est faiblement hyperbolique si et seulement si $X$ est hyperbolique.

Proposition 2.4. Tout espace analytique $X$ holomorphiquement séparé de dimension $n$ est faiblement hyperbolique.

Démonstration. En effet, comme $X$ est holomorphiquement séparé, il existe $g_{1}, \ldots, g_{N} \in \mathcal{O}(X)$ qui séparent les points de $X$. L'application $u$ : $X \rightarrow[-\infty,+\infty[$ définie par

$$
u(x):=\frac{1}{2} \log \left(\left|g_{1}(x)\right|^{2}+\ldots+\left|g_{N}(x)\right|^{2}\right) \quad \text { pour } x \in X
$$

est plurisousharmonique sur $X$. Soit $K \subset X$ un compact, et posons $v:=u-c$ où $c:=\sup _{K} u+1$. Considérons l'ouvert $D:=\{x \in X \mid v(x)<0\}$; on a $K \subset D$. Montrons que $D$ est hyperbolique. Soient $x, y \in D, x \neq y$. Il existe $j \in\{1, \ldots, N\}$ tel que $g_{j}(x) \neq g_{j}(y)$. De plus

$$
\left|g_{j}(z)\right|^{2} \leq\left|g_{1}(z)\right|^{2}+\ldots+\left|g_{N}(z)\right|^{2}<e^{2 c} \quad \forall z \in D .
$$

Il en résulte que

$$
d_{D}(x, y) \geq C_{D}(x, y) \geq \varrho_{\mathbb{D}}\left(e^{-c} g_{j}(x), e^{-c} g_{j}(y)\right)>0,
$$

où $d_{D}$ (resp. $C_{D}$ ) désigne la pseudo-distance de Kobayashi (resp. Carathéodory) et $\varrho_{\mathbb{D}}$ désigne la métrique de Poincaré du disque $\mathbb{D}$. Donc $D$ est Kobayashi-hyperbolique.

Si $f \in \operatorname{Hol}(\mathbb{D}, X) \cap \mathcal{C}(\overline{\mathbb{D}}, X)$ est telle que $f(\partial \mathbb{D}) \subset D$, alors $v \circ f(t)<0$ pour tout $t \in \partial \mathbb{D}$. Comme $v \circ f$ est sousharmonique sur $\mathbb{D}$, le principe du maximum implique que $v \circ f<0$ sur $\mathbb{D}$. Donc $f(\overline{\mathbb{D}}) \subset D$, ce qui démontre que $X$ est faiblement hyperbolique.

THÉORÈme 2.5. Soit $f$ une application méromorphe entre une variété complexe $M$ et un espace analytique faiblement hyperbolique $X$. Alors $f$ est holomorphe. En particulier, si $X$ est une variété faiblement hyperbolique, alors $\operatorname{Aut}(X)=\operatorname{Bim}(X)$.

Démonstration. On peut supposer que $M=\mathbb{D}^{m}$ et les singularités de $f$ sont incluses dans $\{0\} \times \mathbb{D}^{m-1}$. Alors $g=f_{\mid \mathbb{D}^{*} \times \mathbb{D}^{m-1}}$ est holomorphe.

a) Montrons que pour tout $t \in \mathbb{D}^{m-1}$ la fonction $g_{t} \in \operatorname{Hol}\left(\mathbb{D}^{*}, X\right)$, où $g_{t}(z)=f(z, t)$, se prolonge holomorphiquement à $\mathbb{D}$.

Soit $K=f(0, t)$. Comme $f$ est méromorphe, $K$ est compact. Or $X$ est faiblement hyperbolique, donc il existe $\Omega$ hyperbolique qui contient $K$. On 
peut trouver $\varepsilon>0$ tel que $g_{t}\left(\mathbb{D}_{\varepsilon}^{*}\right) \subset \Omega$, sinon on construit une suite $\left(z_{n}\right)_{n}$ de $\mathbb{D}^{*}$ telle que $z_{n} \rightarrow 0$ et $g_{t}\left(z_{n}\right) \notin \Omega$ pour tout $n \geq 0$, d'où $\left\{\lim _{n \rightarrow \infty} f\left(z_{n}, t\right)\right\} \cap$ $\{f(0, t)\}=\emptyset$, ce qui est absurde car $\{f(0, t)\}$ est l'ensemble limite. Comme $f_{\mid \mathbb{D}_{\varepsilon} \times\{t\}}$ est méromorphe et $\Omega$ hyperbolique, $f_{\mid \mathbb{D}_{\varepsilon} \times\{t\}}$ est holomorphe [6], donc $g_{t}$ se prolonge holomorphiquement à $\mathbb{D}$ en $\bar{g}_{t}$.

b) Montrons que pour tout $t \in \mathbb{D}^{m-1}, f$ est holomorphe au point $(0, t)$.

Soient $V$ un voisinage relativement compact de $t$ dans $\mathbb{D}^{m-1}$ et $\left.r \in\right] 0,1[$. Puisque $f_{\left(\mathbb{D}^{*} \times \mathbb{D}^{m-1}\right.}$ est holomorphe, $K=f\left(\partial \mathbb{D}_{r} \times \bar{V}\right)$ est compact. Comme $X$ est faiblement hyperbolique, il existe $\Omega$ hyperbolique qui contient $K$ vérifiant la définition 2.3. Soit $v \in V$. D'après a), $\bar{g}_{v}$ est holomorphe de $\mathbb{D}$ dans $X$ et $\bar{g}_{v}\left(\partial \mathbb{D}_{r}\right)=f\left(\partial \mathbb{D}_{r} \times\{v\}\right) \subset K$, donc $\bar{g}_{v}\left(\mathbb{D}_{r}\right) \subset \Omega$. Alors $f\left(\mathbb{D}_{r} \times\{v\}\right) \subset \Omega$ pour tout $v \in V$, d'où $f\left(\mathbb{D}_{r} \times V\right) \subset \Omega$, et comme $\mathbb{D}_{r} \times V$ est un ouvert de $\mathbb{D}^{m}, f_{\mid \mathbb{D}_{r} \times V}$ est méromorphe. Puisque $\Omega$ est hyperbolique, $f_{\mid \mathbb{D}_{r} \times V}$ est holomorphe, ceci pour tout $t \in \mathbb{D}^{m-1}$. On conclut donc que $f$ est holomorphe de $M$ dans $X$.

3. $\mathbb{D}^{*}$-extension et propriété de prolongement de Hartogs. On démontre ici que si $X$ est faiblement hyperbolique et possède la $\mathbb{D}^{*}$-EP, alors $X$ possède aussi la $(\mathrm{PPH})$.

Proposition 3.1. Soit $X$ un espace analytique complexe faiblement hyperbolique. Si $X$ possède la propriété $\mathbb{D}^{*}$-EP, alors $X$ possède aussi la $(\mathrm{PPH})$.

Démonstration. La démonstration se base sur un théorème de Shiffman $([10])$ : si pour toute suite $\left(f_{n}\right) \subset \operatorname{Hol}(\mathbb{D}, X)$, la convergence de $\left(f_{n \mid \mathbb{D}^{*}}\right)$ dans $\operatorname{Hol}\left(\mathbb{D}^{*}, X\right)$ implique la convergence de $\left(f_{n}\right)$ dans $\operatorname{Hol}(\mathbb{D}, X)$, alors $X$ a la propriété $(\mathrm{PPH})$.

Soit alors $\left(f_{n}\right)_{n \geq 1} \subset \operatorname{Hol}(\mathbb{D}, X)$ convergeant dans $\operatorname{Hol}\left(\mathbb{D}^{*}, X\right)$ vers une application $f \in \operatorname{Hol}\left(\mathbb{D}^{*}, X\right)$. Comme $X$ a la $\mathbb{D}^{*}$-EP, il existe $g \in \operatorname{Hol}(\mathbb{D}, X)$ telle que $g=f$ sur $\mathbb{D}^{*}$. Posons $x_{0}=g(0)$ et $K:=\bar{V}$ où $V \subset \subset X$ est un voisinage de $x_{0}$. Comme $X$ est faiblement hyperbolique, soit $D$ un ouvert hyperbolique contenant $K$ et vérifiant (ii) dans la définition 2.3 .

Soit $r \in] 0,1\left[\right.$. Il existe $n_{0} \geq 1$ tel que $f_{n}\left(\partial \mathbb{D}_{r}\right) \subset V$ pour $n \geq n_{0}$. La condition (ii) dans la définition 2.3 implique que

$$
f_{n}\left(\overline{\mathbb{D}}_{r}\right) \subset D \quad \forall n \geq n_{0} .
$$

Finalement, on note encore par $f_{n}$ l'application $f_{n \mid \mathbb{D}_{r}}$; on a $\left(f_{n}\right)_{n \geq n_{0}} \subset$ $\operatorname{Hol}\left(\mathbb{D}_{r}, D\right)$. On va montrer que la suite $\left(f_{n}\right)_{n}$ converge vers $g$ dans $\operatorname{Hol}\left(\mathbb{D}_{r}, D\right)$.

Comme $D$ est hyperbolique, il suffit de montrer que pour tout $s \in] 0, r[$ et tout $\varepsilon>0$ il existe $N \in \mathbb{N}$ tel que pour tout $n \geq N$,

$$
d_{D}\left(f_{n}(t), g(t)\right)<\varepsilon \quad \forall t \in \overline{\mathbb{D}}(0, s) .
$$

Soient $s \in] 0, r\left[\right.$ et $\varepsilon>0$. Choisissons $\left.s^{\prime} \in\right] 0, s\left[\right.$ de sorte que $d_{\mathbb{D}}(0, t)<\varepsilon / 3$ si 
$|t| \leq s^{\prime}$. Puisque $\left(f_{n \mid \mathbb{D}^{*}}\right)_{n}$ converge vers $f$ sur $\operatorname{Hol}\left(\mathbb{D}^{*}, D\right)$, il suffit de trouver $N \in \mathbb{N}$ tel que pour tout $n \geq N$,

$$
d_{D}\left(f_{n}(t), g(t)\right)<\varepsilon \quad \text { si }|t| \leq s^{\prime} .
$$

Notons qu'il existe $N \in \mathbb{N}$ tel que pour tout $n \geq N$,

$$
d_{D}\left(f_{n}(t), g(t)\right)<\varepsilon / 3 \quad \text { si }|t|=s^{\prime} .
$$

Pour tout $t \in \mathbb{C}$ tel que $|t| \leq s^{\prime}$ soit $\tilde{t}$ tel que $|\widetilde{t}|=s^{\prime}$ et $d_{\mathbb{D}}(t, \widetilde{t})<\varepsilon / 3$. Alors pour tout $n \geq N$ on a

$$
\begin{aligned}
d_{D}\left(f_{n}(t), g(t)\right) & \leq d_{D}\left(f_{n}(t), f_{n}(\widetilde{t})\right)+d_{D}\left(f_{n}(\widetilde{t}), g(\widetilde{t})\right)+d_{D}(g(\widetilde{t}), g(t)) \\
& \leq d_{\mathbb{D}}(t, \widetilde{t})+\varepsilon / 3+d_{\mathbb{D}}(\widetilde{t}, t)<\varepsilon,
\end{aligned}
$$

ce qui démontre que la suite $\left(f_{n}\right)_{n}$ converge vers $g$ dans $\operatorname{Hol}(\mathbb{D}, X)$.

Dans [21], Do Duc Thai et P. J. Thomas ont donné un exemple de variété analytique $X$ (en fait un domaine de $\mathbb{C}^{2}$ ) qui a la propriété $\mathbb{D}^{*}$-EP sans être Kobayashi-hyperbolique, mais $X$ reste quand même faiblement hyperbolique. Notons aussi que dans cet exemple, $X$ est un ouvert pseudoconvexe de $\mathbb{C}^{2}$, donc $X$ possède la (PPH).

Soit $X$ un espace analytique. On dit que $X$ est disque convexe si pour tout compact $K \subset X$, il existe un compact $L \subset X$ tel que pour tout disque analytique $f \in \operatorname{Hol}(\mathbb{D}, X) \cap \mathcal{C}(\overline{\mathbb{D}}, X)$, si $f(\partial \mathbb{D}) \subset K$ alors $f(\overline{\mathbb{D}}) \subset L$.

Il est clair que si $X$ est pseudoconvexe, alors $X$ est disque convexe. Il suffit de prendre $L=\widehat{K}_{P(X)}$, où $P(X)$ est le cône des fonctions plurisousharmoniques continues sur $X$.

Dans [20], Do Duc Thai et Nguyen Le Huong ont démontré que si $X$ est pseudoconvexe, la $\mathbb{D}^{*}$-EP implique la $(\mathrm{PPH})$. Nous obtenons le résultat de [20] comme corollaire de la proposition 3.1.

Corollaire 3.2. Soit $X$ un espace analytique disque convexe. Si $X$ possède la $\mathbb{D}^{*}$-EP, alors $X$ possède aussi la $(\mathrm{PPH})$.

Démonstration. Il suffit de montrer que $X$ est faiblement hyperbolique. Pour cela, considérons $K \subset X$ un compact. Il existe un compact $L \subset X$, qu'on peut choisir contenant $K$, vérifiant :

$$
\forall f \in \operatorname{Hol}(\mathbb{D}, X) \cap \mathcal{C}(\overline{\mathbb{D}}, X), \quad \text { si } f(\partial \mathbb{D}) \subset K \text { alors } f(\overline{\mathbb{D}}) \subset L .
$$

Puisque $X$ ne contient pas de courbe holomorphe, il existe un ouvert $W \subset \subset$ $X$ Kobayashi-hyperbolique tel que $L \subset W$ [5, Theorem 3.6.7, p. 105], ce qui démontre le résultat.

Comme corollaire de la proposition 3.1, on obtient aussi le résultat suivant. 
ThÉORÈme 3.3. Soit $X$ un espace analytique tel que $X$ est un revêtement ramifié d'un espace analytique, holomorphiquement séparé de dimension $n$. Si X possède la $\mathbb{D}^{*}-\mathrm{EP}$, alors $X$ possède la $(\mathrm{PPH})$.

Soient $X, Y$ des espaces analytiques complexes, et $\pi: X \rightarrow Y$ une application holomorphe et à fibre discrète. On dit que $X$ est un domaine de Riemann ramifié au-dessus de $Y$. Lorsque $\pi$ est localement biholomorphe, le domaine de Riemann est dit non ramifié.

Proposition 3.4. Soit $\pi: X \rightarrow M$ un domaine de Riemann non ramifié au-dessus d'une variété de Stein $M$. Si X possède la $\mathbb{D}^{*}-\mathrm{EP}$, alors $X$ est de Stein.

Démonstration. Le théorème 3.3 implique que $X$ possède la $(\mathrm{PPH})$. Donc $X$ est en particulier Hartogs convexe, i.e. que tout plongement holomorphe $\varphi: H_{k}(r) \rightarrow X$ admet un prolongement holomorphe à $\mathbb{D}^{k}$, où $k=\operatorname{dim} X$ et $H_{k}(r)$ est la figure de Hartogs dans $\mathbb{D}^{k}$. Le théorème de Docquier-Grauert [3] implique que $X$ est de Stein.

4. Généralisation du théorème de Picard. Le théorème de Picard dit que toute fonction holomorphe sur $\mathbb{D}^{*}$ ayant une singularité essentielle en 0 ne peut omettre plus d'une valeur dans $\mathbb{C}$. Ce théorème a été généralisé en dimension supérieure par plusieurs auteurs. Dans cette partie, on démontre une telle généralisation. Le résultat que nous obtenons généralise celui de [19].

Avant d'énoncer le résultat, nous rappelons quelques définitions. Soient $U \subset \mathbb{C}^{n}, V \subset \mathbb{C}^{m}$ des domaines et $E \subset U, F \subset V$ des ensembles non pluripolaires. Posons $\Sigma:=(E \times V) \cup(U \times F)$ la croix inscrite dans $U \times V$. On dit que $f: \Sigma \rightarrow X$ est séparément holomorphe si :

(i) $f_{z}=f(z, \cdot): V \rightarrow X$ est holomorphe pour tout $z \in E$,

(ii) $f^{w}=f(\cdot, w): U \rightarrow X$ est holomorphe pour tout $w \in F$.

La fonction extrémale relative associée au couple $(E, U)$ est donnée par $\omega(z, E, U)=\sup \{u(z) \mid u \in \operatorname{Psh}(U), u \leq 0$ sur $E, u \leq 1 \operatorname{sur} U\} \quad(z \in U)$.

La régularisée semi-continue supérieurement $\omega^{*}(\cdot, E, U)$ est plurisousharmonique sur $U$.

ThÉORÈme 4.1. Soient $M$ une variété analytique et $A \subset M$ un sousensemble analytique fermé de $M$ avec codim $A \geq 1$. Soit $Y$ un espace analytique faiblement hyperbolique et possédant la $\mathbb{D}^{*}$-EP. Alors toute application holomorphe $f: M \backslash A \rightarrow Y$ se prolonge en une application holomorphe $F: M \rightarrow Y$.

Démonstration. La démonstration se fait en deux étapes. 
ÉtApe 1 : Les singularités de $A$ sont à croisements normaux. La question étant locale, on peut supposer que $M=\mathbb{D}^{n} \times \mathbb{D}^{l}$ et que $M \backslash A=$ $\left(\mathbb{D}^{*}\right)^{n} \times \mathbb{D}^{l}$. On va montrer que pour tout $p \in \mathbb{N}^{*}$ et tout $q \in \mathbb{N}$ tels que $p+q \leq n+l$, toute application holomorphe $f:\left(\mathbb{D}^{*}\right)^{p} \times \mathbb{D}^{q} \rightarrow Y$ admet un prolongement holomorphe à $\mathbb{D}^{p} \times \mathbb{D}^{q}$. On raisonne par récurrence sur $p$.

Si $p=1$, soit $q \in \mathbb{N}$ tel que $1+q \leq n+l$ et soit $f: \mathbb{D}^{*} \times \mathbb{D}^{q} \rightarrow Y$ holomorphe. Pour tout $z \in \mathbb{D}^{q}, f^{z}:=f(\cdot, z): \mathbb{D}^{*} \rightarrow Y$ est holomorphe et se prolonge en une application holomorphe $\tilde{f}^{z}: \mathbb{D} \rightarrow Y$ du fait que $Y$ possède la $\mathbb{D}^{*}$-EP. Pour tout $t \in \mathbb{D}^{*}, f_{t}:=f(t, \cdot): \mathbb{D}^{q} \rightarrow Y$ est holomorphe. Posons $\Sigma:=\left(\mathbb{D}^{*} \times \mathbb{D}^{q}\right) \cup\left(\mathbb{D} \times \mathbb{D}^{q}\right)$ et considérons $g: \Sigma \rightarrow Y$ définie par

$$
g(t, z):= \begin{cases}f(t, z) & \text { si }(t, z) \in \mathbb{D}^{*} \times \mathbb{D}^{q} \\ \widetilde{f}^{z}(t) & \text { si }(t, z) \in \mathbb{D} \times \mathbb{D}^{q}\end{cases}
$$

L'application $g$ ainsi définie est séparément holomorphe sur $\Sigma$. Comme $Y$ possède la $(\mathrm{PPH})$ (découle de la proposition 3.1), d'après [1] il existe une application holomorphe $\widetilde{g}: \widetilde{\Sigma} \rightarrow Y$ telle que $\widetilde{g}=g$ sur $\widetilde{\Sigma} \cap \Sigma$, avec

$$
\widetilde{\Sigma}=\left\{(t, z) \in \mathbb{D} \times \mathbb{D}^{q} \mid \omega^{*}\left(t, \mathbb{D}^{*}, \mathbb{D}\right)+\omega^{*}\left(z, \mathbb{D}^{q}, \mathbb{D}^{q}\right)<1\right\}=\mathbb{D} \times \mathbb{D}^{q} .
$$

Ainsi $g$ est holomorphe sur $\mathbb{D} \times \mathbb{D}^{q}$ et $g=f$ sur $\mathbb{D}^{*} \times \mathbb{D}^{q}$.

Supposons le résultat vrai pour $p \geq 1$; montrons-le pour $p+1$. Soit alors $q \in \mathbb{N}$ tel que $p+1+q \leq n+l$ et soit $f:\left(\mathbb{D}^{*}\right)^{p+1} \times \mathbb{D}^{q} \rightarrow Y$ holomorphe.

Écrivons $\left(\mathbb{D}^{*}\right)^{p+1} \times \mathbb{D}^{q}=\mathbb{D}^{*} \times\left(\left(\mathbb{D}^{*}\right)^{p} \times \mathbb{D}^{q}\right)$. Alors pour tout $t \in \mathbb{D}^{*}$ l'application $f_{t}:=f(t, \cdot):\left(\mathbb{D}^{*}\right)^{p} \times \mathbb{D}^{q} \rightarrow Y$ est holomorphe. Comme $p+q$ $\leq p+1+q \leq n+l$, l'hypothèse de récurrence implique qu'il existe une application holomorphe $\widetilde{f}_{t}: \mathbb{D}^{p} \times \mathbb{D}^{q} \rightarrow Y$ qui prolonge $f_{t}$. Pour tout $z \in$ $\left(\mathbb{D}^{*}\right)^{p} \times \mathbb{D}^{q}$ l'application $f^{z}:=f(\cdot, z): \mathbb{D}^{*} \rightarrow Y$ est holomorphe, et comme $Y$ possède la $\mathbb{D}^{*}$-EP, il existe $\tilde{f}^{z}: \mathbb{D} \rightarrow Y$ holomorphe qui prolonge $f^{z}$. Posons $\Sigma:=\left(\mathbb{D}^{*} \times \mathbb{D}^{p+q}\right) \cup\left(\mathbb{D} \times\left(\left(\mathbb{D}^{*}\right)^{p} \times \mathbb{D}^{q}\right)\right)$ et soit $g: \Sigma \rightarrow Y$ donnée par

$$
g(t, z):= \begin{cases}\widetilde{f}_{t}(z) & \text { si }(t, z) \in \mathbb{D}^{*} \times \mathbb{D}^{p+q}, \\ \widetilde{f}^{z}(t) & \text { si }(t, z) \in \mathbb{D} \times\left(\left(\mathbb{D}^{*}\right)^{p} \times \mathbb{D}^{q}\right) .\end{cases}
$$

Ainsi définie, l'application $g$ est séparément holomorphe sur $\Sigma$. D'après [1] il existe $\widetilde{g}: \widetilde{\Sigma} \rightarrow Y$ holomorphe qui prolonge $g$. Or $\widetilde{\Sigma}=\mathbb{D}^{p+1} \times \mathbb{D}^{q}$, on a donc le résultat souhaité. Pour $p=n$ et $q=l$ on termine l'étape 1 .

ÉtAPe 2 : Cas général. Par le théorème de désingularisation de Hironaka, il existe une variété analytique $N$ et un sous-ensemble analytique $B \subset N$ avec $\operatorname{codim} B \geq 1$ à croisements normaux et une application holomorphe propre et surjective $\pi: N \rightarrow M$ telle que $B=\pi^{-1}(A)$. On définit $g: N \backslash B \rightarrow Y$ par $g:=f \circ \pi$. L'étape 1 implique que $g$ a un prolongement holomorphe $G: N \rightarrow Y$ telle que $G=g$ sur $N \backslash B$. Comme $\pi$ est une modification propre, $\pi^{-1}$ définie une application méromorphe de $M$ sur $N$. Ainsi, $F:=G \circ \pi^{-1}$ définit une application méromorphe de $M$ sur $Y$. Le 
théorème 2.5 implique que $F$ se prolonge en une application holomorphe $\widetilde{F}: M \rightarrow Y$, ce qui démontre le théorème.

Comme conséquence de ce théorème, nous obtenons le résultat suivant.

ThÉORÈme 4.2. Soit $M$ une variété analytique complexe avec $\operatorname{dim} M=$ $m \geq 1$, et soit $A \subset M$ un sous-ensemble fermé de $(2 m-2)$-mesure de Hausdorff localement finie. Soit $Y$ un espace analytique faiblement hyperbolique possèdant la $\mathbb{D}^{*}$-EP. Alors toute application holomorphe $f: M \backslash A \rightarrow Y$ admet un prolongement holomorphe à $M$.

Soit $A^{\prime}$ l'ensemble des points $a \in A$ au voisinages desquels $f$ admet un prolongement holomorphe. Alors $S=A \backslash A^{\prime}$ est fermé. Comme $M \backslash A$ est localement connexe, le prolongement holomorphe local de $f$ permet de définir une application holomorphe unique, qu'on note encore $f, f: M \backslash S \rightarrow Y$.

La démonstration du théorème 4.2 découle des lemmes suivants.

LEMME 4.3. L'ensemble $S$ est localement pseudoconcave dans $M$.

Démonstration. Soient $a \in S, V$ un voisinage de Stein de $a$, et $\varphi$ : $H_{m}(r) \rightarrow V \backslash S$ un plongement holomorphe. Comme $V$ est de Stein, il existe un plongement holomorphe $\widetilde{\varphi}: \mathbb{D}^{m} \rightarrow V$. Or $Y$ possède la $(\mathrm{PPH})$, $f \circ \varphi: H_{m}(r) \rightarrow Y$ admet un prolongement holomorphe $g: \mathbb{D}^{m} \rightarrow Y$, et par suite $f$ s'étend holomorphiquement à $\widetilde{\varphi}\left(\mathbb{D}^{m}\right) \subset V$. Par définition de $S$, on a $\widetilde{\varphi}\left(\mathbb{D}^{m}\right) \subset V \backslash S$, donc $V \backslash S$ est Hartogs convexe. Puisque $V$ est de Stein, $V \backslash S$ l'est aussi d'après [3].

LEMME 4.4. Soit $S \subset M$ non vide, localement pseudoconcave et de $(2 m-2)$-mesure de Hausdorff localement finie, où $m=\operatorname{dim} M$. Alors $S$ est un sous-ensemble analytique de dimension $m-1$ de $M$.

Démonstration. La question étant locale, on peut supposer que $M$ est un domaine $D \subset \mathbb{C}^{m}$. Soit $a \in S$; par un changement de coordonnées on peut supposer que $a=0$ et que $S \cap\left\{z \in D \mid z_{1}=\ldots=z_{m-1}=0\right\}$ est dénombrable. Il existe alors un voisinage $V:=V^{\prime} \times V^{\prime \prime} \subset \mathbb{C}^{m-1} \times \mathbb{C}$ de 0 tel que la projection

$$
\Pi: S \cap V \rightarrow V^{\prime} \quad \text { soit propre. }
$$

Il en résulte que les fibres $S \cap V \cap\left\{\left(z^{\prime}, z_{m}\right) \in V \mid z^{\prime}=c^{\prime}\right\}$ sont finies pour presque tout $c^{\prime} \in V^{\prime}$. Le théorème d'Oka-Nishino ([9]) implique que $S \cap V$ est un sous-ensemble analytique de dimension $m-1$.

5. Hyperbolicité et théorème de Hartogs. Dans cette partie, on donne une généralisation du théorème de Hartogs pour les applications séparément holomorphes à valeurs dans les espaces faiblement hyperboliques. Nous commençons par rappeler un résultat de Shiffman [12], qui nous sera très utile dans ce qui suit. 
ThÉORÈme 5.1 ([12, Théorème 1]). Soient $U \subset \mathbb{C}^{n}, V \subset \mathbb{C}^{m}$ deux domaines et $K \subset \mathbb{C}^{m}$ un compact connexe contenant $V$. Soit $f: U \times V \rightarrow X$ une application holomorphe. Si $f_{z}$ a un prolongement holomorphe à $K$ pour tout $z \in U$, alors il existe un sous-ensemble pluripolaire fermé $P \subset U$ et une application holomorphe $\widetilde{f}:(U \backslash P) \times K \rightarrow X$ telle que $\widetilde{f}=f$ sur $(U \backslash P) \times V$.

Une application $f: U \times V \rightarrow X$ sera dite séparément holomorphe si elle l'est sur la croix $\Sigma=(U \times V) \cup(U \times V)$.

Lemme 5.2. Soient $U \subset \mathbb{C}^{n}, V \subset \mathbb{C}^{m}$ deux domaines et $X$ un espace analytique hyperbolique. Si $f: U \times V \rightarrow X$ est une application séparément holomorphe, alors $f$ est holomorphe sur $U \times V$.

Démonstration. Il suffit de montrer que $f$ est continue car dans ce cas on se ramène localement à l'espace euclidien.

Soient $(a, b) \in U \times V$ et $\left(\left(z_{n}, w_{n}\right)\right)_{n} \subset U \times V$ tels que $\left(z_{n}, w_{n}\right) \rightarrow(a, b)$. Alors il existe $r>0$ et $N \in \mathbb{N}$ tels que pour tout $n \geq N$ on a $\left(z_{n}, w_{n}\right) \in$ $\Delta(a, r) \times \Delta(b, r)$, où $\Delta(a, r)$ (resp. $\Delta(b, r)$ ) est le polydisque centré en $a$ inclus dans $U$ (resp. le polydisque centré en $b$ inclus dans $V$ ). Pour $n \geq N$, on a

$$
\begin{aligned}
d_{X}\left(f(a, b), f\left(z_{n}, w_{n}\right)\right) & \leq d_{X}\left(f(a, b), f\left(a, w_{n}\right)\right)+d_{X}\left(f\left(a, w_{n}\right), f\left(z_{n}, w_{n}\right)\right) \\
& \leq d_{\Delta(b, r)}\left(b, w_{n}\right)+d_{\Delta(a, r)}\left(a, z_{n}\right)
\end{aligned}
$$

car $f_{a} \in \operatorname{Hol}(\Delta(b, r), X)$ et $f^{w_{n}} \in \operatorname{Hol}(\Delta(a, r), X)$. Comme $\Delta(a, r)$ et $\Delta(b, r)$ sont hyperboliques, $d_{\Delta(a, r)}\left(a, z_{n}\right) \rightarrow 0$ et $d_{\Delta(b, r)}\left(b, w_{n}\right) \rightarrow 0$, d'où $f$ est continue au point $(a, b)$.

ThÉorème 5.3. Soient $U \subset \mathbb{C}^{n}, V \subset \mathbb{C}^{m}$ deux domaines et $X$ un espace analytique faiblement hyperbolique. Si $f: U \times V \rightarrow X$ est séparément holomorphe, alors $f$ est holomorphe.

Démonstration (par récurrence sur $n$ ). Supposons que $n=1$.

(a) D'après [1], il existe des domaines $\emptyset \neq U_{0} \subset U$ et $\emptyset \neq V_{0} \subset V$ tels que $f: U_{0} \times V_{0} \rightarrow X$ est holomorphe. Quitte à prendre un sous-domaine simplement connexe on peut supposer que $U_{0}$ est simplement connexe.

Soit $V_{1}$ un domaine relativement compact de $V$ qui contient $V_{0}$. Comme $f_{z} \in \operatorname{Hol}\left(\bar{V}_{1}, X\right)$ pour tout $z \in U_{0}$, le théorème 5.1 implique qu'il existe $E \subset U_{0}$ polaire fermé tel que $f:\left(U_{0} \backslash E\right) \times \bar{V}_{1} \rightarrow X$ est holomorphe.

Soit $U_{1} \subset U_{0}$ un domaine relativement compact. Il existe un domaine $D$ simplement connexe tel que $\bar{D} \subset U_{0}, U_{1} \subset D, \partial D \cap E=\emptyset$ et $\partial D$ est une courbe de Jordan fermée. Soit $\chi: \mathbb{D} \rightarrow D$ une application biholomorphe, où $\mathbb{D}$ est le disque unité de $\mathbb{C}$. Comme $\partial D$ est une courbe de Jordan fermée, $\chi$ se prolonge en un homéomorphisme de $\overline{\mathbb{D}}$ dans $\bar{D}$ (théorème de OsgoodCarathéodory). 
Soit $K=f\left(\partial D \times \bar{V}_{1}\right)$. Il existe $\Omega$ hyperbolique qui contient $K$ et vérifie la définition 2.3 .

On a $f^{w} \circ \chi \in \operatorname{Hol}(\mathbb{D}, X) \cap \mathcal{C}(\overline{\mathbb{D}}, X)$ pour tout $w \in V_{1}$ et $f^{w} \circ \chi(\partial \mathbb{D})=$ $f(\partial D \times\{w\}) \subset K$, donc $f^{w} \circ \chi(\mathbb{D}) \subset \Omega$, d'où $f^{w}(D) \subset \Omega$, et ceci pour tout $w \in V_{1}$, ce qui implique $f\left(D \times V_{1}\right) \subset \Omega$. Par suite, d'après le lemme 5.2, $f \in \operatorname{Hol}\left(U_{1} \times V_{1}, X\right)$, ceci indépendamment de $U_{1}$ (resp. $V_{1}$ ) dans $U_{0}$ (resp. $V)$, donc $f \in \operatorname{Hol}\left(U_{0} \times V, X\right)$.

(b) Soit $U_{1}$ un domaine relativement compact de $U$ qui contient $U_{0}$. D'après (a) on a $f \in \operatorname{Hol}\left(U_{0} \times V, X\right)$ et $f^{w} \in \operatorname{Hol}\left(\bar{U}_{1}, X\right)$ pour tout $w \in V$. Donc il existe $P \subset V$ pluripolaire fermé tel que $f \in \operatorname{Hol}\left(\bar{U}_{1} \times(V \backslash P), X\right)$. Soient $V_{2} \subset V \backslash P$ un domaine relativement compact et $V_{1}$ un domaine relativement compact de $V$ qui contient $V_{2}$. On a $f \in \operatorname{Hol}\left(U_{1} \times V_{2}, X\right)$ et $f_{z} \in \operatorname{Hol}\left(\bar{V}_{1}, X\right)$ pour tout $z \in U_{1}$, donc il existe $F$ polaire fermé dans $U_{1}$ tel que $f:\left(U_{1} \backslash F\right) \times \bar{V}_{1} \rightarrow X$ est holomorphe. On reprend la même démonstration que dans (a) et on montre que $f \in \operatorname{Hol}\left(U_{1} \times V_{1}, X\right)$. Comme $U_{1}$ (resp. $V_{1}$ ) est arbitraire dans $U$ (resp. $V$ ), on a $f \in \operatorname{Hol}(U \times V, X)$.

Supposons maintenant le résultat vrai pour $n-1$. Soit $V_{1}$ un domaine relativement compact de $V$. Il existe $P \subset U$ pluripolaire fermé tel que $f: U \backslash P \times \bar{V}_{1} \rightarrow X$ est holomorphe. Soit $a \in P$. Il existe $r>0$ tel que $\Delta_{a}(r) \times V_{1} \subset U \times V$. On montre comme précédemment que $f \in$ $\operatorname{Hol}\left(\Delta_{a}(r) \times V_{1}\right)$ (en utilisant l'hypothèse de récurrence), ce qui achève la démonstration.

T. Terada a affaibli les hypothèses sur l'un des facteurs dans le théorème de Hartogs, obtenant ainsi une généralisation de ce théorème pour les fonctions séparement holomorphes. Dans ce qui suit on montre que ce résultat reste vrai lorsqu'on remplace $\mathbb{C}$ par un espace hyperbolique ou un espace holomorphiquement séparé de dimension pure.

Lemme 5.4. Soient $U \subset \mathbb{C}^{n}, V \subset \mathbb{C}^{m}$ deux domaines, $\emptyset \neq W \subset V$ un ouvert et $X$ un espace hyperbolique. Soit $f: U \times V \rightarrow X$ une application telle que:

(i) $f: U \times W \rightarrow X$ est holomorphe,

(ii) $f_{z} \in \operatorname{Hol}(V, X)$ pour tout $z \in U$.

Alors $f$ est holomorphe sur $U \times V$.

Démonstration. Montrons par récurrence sur $n$ que $f \in \operatorname{Hol}(U \times V, X)$.

(a) Cas où $n=1$. Soit $V_{1}$ un domaine relativement compact de $V$ tel que $V_{0}:=V_{1} \cap W \neq \emptyset$; alors d'après le théorème 5.1 , il existe $P \subset U$ polaire fermé tel que $f \in \operatorname{Hol}\left((U \backslash P) \times \bar{V}_{1}, X\right)$.

Soit $D$ un ouvert relativement compact de $U$ tel que $\partial D \cap P=\emptyset$. Puisque $P$ est polaire fermé, $D \cap P$ est compact. Soient $U_{1} \subset D$ un domaine rela- 
tivement compact tel que $\bar{U}_{1} \cap P=D \cap P$ et $M=\left\{w \in V_{1} \mid f\right.$ holomorphe au voisinage de $\left.\bar{U}_{1} \times\{w\}\right\}$. On a $V_{0} \subset M$, donc $M$ est un ouvert non vide de $V_{1}$.

Soit $w \in M$; alors $f^{w} \in \operatorname{Hol}(U \backslash P, X)$ et $f^{w} \in \operatorname{Hol}\left(\bar{U}_{1}, X\right)$. Il en résulte que $f^{w} \in \operatorname{Hol}(D, X)$.

Soient $(a, b) \in D \times\left(\bar{M} \cap V_{1}\right),\left(\left(a_{n}, b_{n}\right)\right)_{n} \subset D \times\left(\bar{M} \cap V_{1}\right)$ et $\left(w_{n}\right)_{n} \subset M$ tels que $\left(a_{n}, b_{n}\right) \rightarrow(a, b)$ et $w_{n} \rightarrow b$. Alors on a

$$
\begin{aligned}
d_{X}(f(a, b), f( & \left.\left.a_{n}, b_{n}\right)\right) \\
\leq & d_{X}\left(f(a, b), f\left(a, b_{n}\right)\right)+d_{X}\left(f\left(a, b_{n}\right), f\left(a_{n}, b_{n}\right)\right) \\
\leq & d_{V_{1}}\left(b, b_{n}\right)+d_{X}\left(f\left(a, w_{n}\right), f\left(a, b_{n}\right)\right) \\
& +d_{X}\left(f\left(a, w_{n}\right), f\left(a_{n}, w_{n}\right)\right)+d_{X}\left(f\left(a_{n}, w_{n}\right), f\left(a_{n}, b_{n}\right)\right) \\
\leq & d_{V_{1}}\left(b, b_{n}\right)+d_{V_{1}}\left(w_{n}, b_{n}\right)+d_{D}\left(a, a_{n}\right)+d_{V_{1}}\left(w_{n}, b_{n}\right),
\end{aligned}
$$

qui tend vers 0 . Donc $f$ est continue de $D \times\left(\bar{M} \cap V_{1}\right)$ dans $X$.

Supposons que $\partial M \cap V_{1} \neq \emptyset$. Soit $b \in \partial M \cap V_{1}$ et soit $a \in \bar{U}_{1}$. Comme $f$ est continue, il existe un ouvert de Stein $X_{0} \subset X$ et $\varepsilon, \eta$ tels que $B(a, \varepsilon) \subset D$, $B(b, 2 \eta) \subset V_{1}$ et $f(B(a, \varepsilon) \times(B(b, 2 \eta) \cap \bar{M})) \subset X_{0}$. Soit $\Gamma$ une composante connexe de $M \cap B(b, \eta)$. On a $\partial \Gamma \cap B(b, \eta) \neq \emptyset$, car sinon on aura $\Gamma=B(b, \eta)$ et donc $b \in M$. De plus on a $\partial \Gamma \cap B(b, \eta) \subset \partial M$. Soit $b_{1} \in \partial \Gamma \cap B(b, \eta)$ et $K=\bar{\Gamma} ; K$ est un compact connexe d'intérieur non vide.

Soit $b_{0} \in \Gamma$. Comme $f$ est holomorphe au point $\left(a, b_{0}\right)$, il existe $\varepsilon_{1}$, $\eta_{0}$ tels que $B\left(b_{0}, \eta_{0}\right) \subset K$ et $f \in \operatorname{Hol}\left(B\left(a, \varepsilon_{1}\right) \times B\left(b_{0}, \eta_{0}\right), X\right)$. En posant $\varepsilon_{0}=\min \left(\varepsilon, \varepsilon_{1}\right)$, on a $f\left(B\left(a, \varepsilon_{0}\right) \times K\right) \subset X_{0}$. Finalement, $f: B\left(a, \varepsilon_{0}\right) \times$ $B\left(b_{0}, \eta_{0}\right) \rightarrow X_{0}$ est holomorphe et pour tout $z \in B\left(a, \varepsilon_{0}\right), f_{z} \in \operatorname{Hol}\left(V_{z}, X_{0}\right)$ où $V_{z}=f_{z}^{-1}\left(X_{0}\right) \supseteq K$. Le théorème 5.1 implique qu'il existe un ensemble $S \subset B\left(a, \varepsilon_{0}\right)$ polaire fermé tel que $f:\left(B\left(a, \varepsilon_{0}\right) \backslash S\right) \times K \rightarrow X_{0}$ soit holomorphe. Comme $X_{0}$ possède la propriété de prolongement de Hartogs, il en résulte, d'après [12], que $f$ est holomorphe sur $B\left(a, \varepsilon_{0}\right) \times K$, donc au point $\left(a, b_{1}\right)$, et ceci pour tout $a \in \bar{U}_{1}$ qui est compact. Donc $f$ est holomorphe au voisinage de $\bar{U}_{1} \times\left\{b_{1}\right\}$, par conséquent $b_{1} \in M$, ce qui est absurde. Ceci implique que $\partial M \cap V_{1}=\emptyset$. Comme $V_{1}$ est connexe, on a $M=V_{1}$ et par suite $f \in \operatorname{Hol}\left(U_{1} \times V_{1}, X\right)$; puisque $U_{1}$ est arbitraire dans $D$, qui est lui aussi arbitraire dans $U$, on a $f \in \operatorname{Hol}\left(U \times V_{1}, X\right)$. Comme $V_{1}$ est aussi arbitraire, $f$ est holomorphe sur $U \times V$.

(b) Supposons que la propriété est vraie jusqu'à l'ordre $n-1$. Soit $V_{1} \subset \subset$ $V$ un domaine relativement compact tel que $V_{0}:=V_{1} \cap W \neq \emptyset$. Il existe $P \subset U$ pluripolaire fermé tel que $f \in \operatorname{Hol}\left((U \backslash P) \times \bar{V}_{1}, X\right)$.

Soit $a \in P$. Alors il existe $r>0$ tel que $\Delta_{a}(r) \times V_{1} \subset U \times V$. On montre comme précédemment que $f \in \operatorname{Hol}\left(\Delta_{a}(r) \times V_{1}\right)$ (en utilisant l'hypothèse de récurrence), ce qui achève la démonstration. 
Proposition 5.5. Soient $U \subset \mathbb{C}^{n}, V \subset \mathbb{C}^{m}$ deux domaines, $E \subset U$ un ensemble non pluripolaire et $X$ un espace hyperbolique. Soit $f: U \times V \rightarrow X$ une application telle que:

(i) $f_{z} \in \operatorname{Hol}(V, X)$ pour tout $z \in E$.

(ii) $f^{w} \in \operatorname{Hol}(U, X)$ pour tout $w \in V$.

Alors $f$ est holomorphe sur $U \times V$.

Démonstration. D'après [1] il existe deux domaines $\emptyset \neq U_{0} \subset U$ et $\emptyset \neq V_{0} \subset V$ tels que $f: U_{0} \times V_{0} \rightarrow X$ soit holomorphe et $U_{0} \cap E=E_{0}$ non pluripolaire. Soit $V_{1} \subset V$ un domaine relativement compact contenant $V_{0}$ et soit l'ensemble $G=\left\{z \in U_{0} \mid f_{z} \in \operatorname{Hol}\left(\bar{V}_{1}, X\right)\right\}$; alors $G$ est non pluripolaire car $E_{0} \subset G$. De plus l'ensemble $A=\{z \in G \mid f$ ne se prolonge pas holomorphiquement au voisinage de $\left.\{z\} \times \bar{V}_{1}\right\}$ est pluripolaire fermé d'après [1, Proposition 2.2] (il suffit de reprendre la démonstration donnée par Shiffman dans [12, Théorème 1]). Il est facile de vérifier que $W=G \backslash A$ est un ouvert; on a alors $f \in \operatorname{Hol}\left(W \times V_{1}, X\right)$.

Comme $f^{w} \in \operatorname{Hol}\left(U_{0}, X\right)$ pour tout $w \in V_{1}$, le lemme 5.4 implique $f \in$ $\operatorname{Hol}\left(U_{0} \times V_{1}, X\right)$. Puisque $V_{1}$ est arbitraire dans $V$, on a $f \in \operatorname{Hol}\left(U_{0} \times V, X\right)$. De même, puisque $f^{w} \in \operatorname{Hol}(U, X)$ pour tout $w \in V$ et $f \in \operatorname{Hol}\left(U_{0} \times V, X\right)$, le lemme 5.4 implique que $f \in \operatorname{Hol}(U \times V, X)$.

ThÉorème 5.6. Soient $U \subset \mathbb{C}^{n}, V \subset \mathbb{C}^{m}$ deux domaines, $E \subset U$ un ensemble non pluripolaire et $X$ un espace analytique holomorphiquement séparé de dimension pure. Soit $f: U \times V \rightarrow X$ une application telle que:

(i) $f_{z} \in \operatorname{Hol}(V, X)$ pour tout $z \in E$,

(ii) $f^{w} \in \operatorname{Hol}(U, X)$ pour tout $w \in V$.

Alors $f$ est holomorphe sur $U \times V$.

Démonstration. Comme $X$ est holomorphiquement séparé de dimension pure, il existe $g \in \operatorname{Hol}\left(X, \mathbb{C}^{N}\right)$ qui sépare les points de $X$. Donc $g \circ f_{z} \in$ $\operatorname{Hol}\left(V, \mathbb{C}^{N}\right)$ pour tout $z \in E$ et $g \circ f^{w} \in \operatorname{Hol}\left(U, \mathbb{C}^{N}\right)$ pour tout $w \in V$. Par conséquent $g \circ f \in \operatorname{Hol}\left(U \times V, \mathbb{C}^{N}\right)$. Soient

$$
\begin{aligned}
\vartheta(x) & :=\frac{1}{2} \log \left(\left|g_{1} \circ f(x)\right|^{2}+\ldots+\left|g_{N} \circ f(x)\right|^{2}\right), \\
\phi(x) & :=\frac{1}{2} \log \left(\left|g_{1}(x)\right|^{2}+\ldots+\left|g_{N}(x)\right|^{2}\right) .
\end{aligned}
$$

On a $\vartheta \in \operatorname{PSH}(U \times V)$ et $\phi \in \operatorname{PSH}(X)$. Soient $U_{1}$ (resp. $V_{1}$ ) des ouverts relativement compacts de $U$ (resp. de $V$ ) avec $U_{1} \cap E$ non pluripolaire. Posons $r:=\sup _{\bar{U}_{1} \times \bar{V}_{1}} \vartheta, \varphi:=\phi-r$ et $\Omega:=\{x \in X \mid \varphi(x)<0\}$. L'ensemble $\Omega$ est hyperbolique (voir la démonstration de la proposition 2.4) et $f\left(U_{1} \times V_{1}\right) \subset \Omega$ (facile à vérifier), donc d'après la proposition 5.5 on a $f \in \operatorname{Hol}\left(U_{1} \times V_{1}, X\right)$. 
Comme $U_{1}$ et $V_{1}$ sont arbitraires, on conclut que $f$ est holomorphe sur $U \times V$.

Remerciements. Les auteurs sont très reconnaissants au referee pour ses suggestions et remarques utiles, qui nous ont permis d'améliorer la rédaction de ce manuscrit.

\section{Références}

[1] O. Alehyane, Une extension du théorème de Hartogs pour les applications séparément holomorphes, C. R. Acad. Sci. Paris Sér. I 324 (1997), 149-152.

[2] O. Alehyane et A. Zeriahi, Une nouvelle version du théorème de Hartogs pour les applications séparément holomorphes entre espaces analytiques, Ann. Polon. Math. 76 (2001), 245-278.

[3] F. Docquier und H. Grauert, Levisches Problem und Rungescher Satz für teilgebiete Steinscher Männigfaltigkeiten, Math. Ann. 140 (1963), 94-123.

[4] S. M. Ivashkovich, The Hartogs phenomenon for holomorphically convex Kähler manifolds, Math. USSR-Izv. 29 (1987), 225-232.

[5] S. Kobayashi, Hyperbolic Complex Spaces, Grundlehren Math. Wiss., Springer, 1998.

[6] A. Kodama, On bimeromorphic automorphisms of hyperbolic complex spaces, Nagoya Math. J. 73 (1979), 1-5.

[7] M. Kwack, Generalization of the big Picard theorem, Ann. of Math. 90 (1969), 9-22.

[8] Nguyen Thanh Van et A. Zeriahi, Une extension du théorème de Hartogs sur les fonctions séparément analytiques, dans : Analyse Complexe Multivariable : Récents Développements (Guadeloupe, 1988), A. Meril (éd.), EditEl, Rende, 1991, 183-194.

[9] T. Nishino, Sur les ensembles pseudoconcaves, J. Math. Kyoto Univ. 1 (1962), 225245.

[10] B. Shiffman, Extension of holomorphic maps into hermitian manifolds, Math. Ann. 194 (1971), 249-258.

[11] —, Separate analyticity and Hartogs theorems, Indiana Univ. Math. J. 38 (1989), 943-957.

[12] - Hartogs theorems for separately holomorphic mappings into complex spaces, C. R. Acad. Sci. Paris 310 (1990), 89-94.

[13] I. Shimoda, Notes on the functions of two complex variables, J. Gakugei Tokushima Univ. 8 (1957), 1-3.

[14] N. Sibony, A class of hyperbolic manifolds, dans : Ann. of Math. Stud. 100, Princeton Univ. Press, 1981, 357-372.

[15] J. Siciak, Analyticity and separate analyticity of functions defined on lower dimensional subsets of $\mathbb{C}^{n}$, Zeszyty Nauk. Uniw. Jagiell. Prace Mat. 13 (1969), 53-70.

[16] - Separately analytic functions and envelopes of holomorphy of some lower dimensional subsets of $\mathbb{C}^{n}$, Ann. Polon. Math. 22 (1970), 145-171.

[17] T. Terada, Sur une certaine condition sous laquelle une fonction de plusieurs variables complexes est holomorphe. Diminution de la condition dans le théorème de Hartogs, Publ. Res. Inst. Math. Sci. Ser. A 2 (1967), 383-396.

[18] D. D. Thai, On the $\mathbb{D}^{*}$-extension and the Hartogs extension, Ann. Scuola Norm. Sup. Pisa 18 (1991), 13-38.

[19] -, $\mathbb{D}^{*}$-extension property and generalization of the big Picard theorem, Vietnam J. Math. 23 (1995), 163-170. 
[20] D. D. Thai and N. L. Huong, On the disc-convexity of complex Banach manifolds, Ann. Polon. Math. 69 (1998), 1-11.

[21] D. D. Thai and P. J. Thomas, $\mathbb{D}^{*}$-extension property without hyperbolicity, Indiana Univ. Math. J. 47 (1998), 1125-1130.

[22] V. P. Zahariuta, Separately holomorphic functions, generalisation of Hartogs theorem and envelopes of holomorphy, Math. USSR-Sb. 30 (1976), 51-76.

Département de Mathématiques

Faculté des Sciences

Université Chouaib Doukkali

B.P. 20, El Jadida, Maroc

E-mail: alehyane@ucd.ac.ma
Département de Mathématiques

Faculté des Sciences

Université Mohammed V

B.P 1014, Rabat, Maroc

E-mail: hichameamal@hotmail.com

Reçu par la Rédaction le 17.12.2001

Révisé le 6.12.2002 